\title{
VR Natural Walking in Impossible Spaces
}

\author{
Daniel Lochner \\ University of Cape Town \\ South Africa \\ daniel@daniellochner.com
}

\author{
James Gain \\ University of Cape Town \\ South Africa \\ jgain@cs.uct.ac.za
}

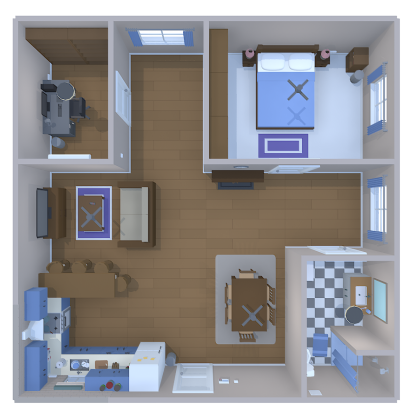

(a) Teleportation

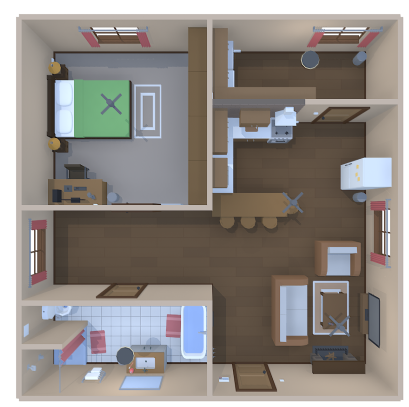

(b) Touchpad/Joystick

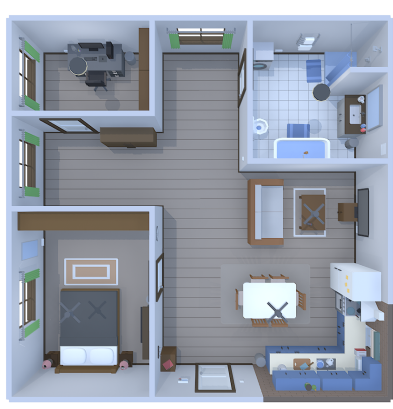

(c) Arm-swinging

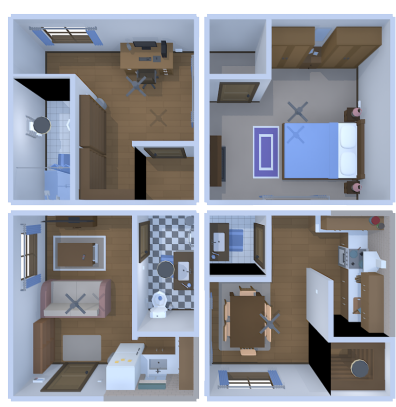

(d) Natural walking

Figure 1: A top-down view of the four testing environments used in our comparative VR locomotion experiment.

\begin{abstract}
Locomotion techniques in Virtual Reality (VR) are the means by which users traverse a Virtual Environment (VE) and are considered an integral and indispensable part of user interaction.

This paper investigates the potential that natural walking in impossible spaces provides as a viable locomotion technique in VR when compared to conventional alternatives, such as teleportation, arm-swinging and touchpad/joystick. In this context, impossible spaces are locally Euclidean orbit-manifolds - subspaces separated by portals that are individually consistent but are able to impossibly overlap in space without interacting.

A quantitative user experiment was conducted with $n=25$ participants, who were asked to complete a set of tasks inside four houses, in each case using a different locomotion technique to navigate. After completing all tasks for a given house, participants were then asked to complete a set of three questionnaires regarding the technique used, namely the Simulator Sickness Questionnaire (SSQ), Game Experience Questionnaire (GEQ) and System Usability Scale (SUS). Time for task completion was also recorded.

It was found that natural walking in impossible spaces significantly improves $(\alpha=0.05)$ immersion (as compared to teleportation and touchpad/joystick, $r>0.7$ ) and system usability (over touch$\mathrm{pad} /$ joystick and arm-swinging, $r \geq 0.38$ ), but seems to lead to slower task completion.
\end{abstract}

Permission to make digital or hard copies of all or part of this work for personal or classroom use is granted without fee provided that copies are not made or distributed for profit or commercial advantage and that copies bear this notice and the full citation on the first page. Copyrights for components of this work owned by others than ACM must be honored. Abstracting with credit is permitted. To copy otherwise, or republish, to post on servers or to redistribute to lists, requires prior specific permission and/or a fee. Request permissions from permissions@acm.org.

MIG '21, November 10-12, 2021, Virtual Event, Switzerland

(c) 2021 Association for Computing Machinery.

ACM ISBN 978-1-4503-9131-3/21/11 . \$15.00

https://doi.org/10.1145/3487983.3488305

\section{CCS CONCEPTS}

- Computing methodologies $\rightarrow$ Virtual reality.

\section{KEYWORDS}

Virtual Reality, locomotion, simulator sickness, redirected walking

ACM Reference Format:

Daniel Lochner and James Gain. 2021. VR Natural Walking in Impossible Spaces. In Motion, Interaction and Games (MIG '21). ACM, New York, NY, USA, 9 pages. https://doi.org/10.1145/3487983.3488305

\section{INTRODUCTION}

Virtual Reality (VR) is regarded as one of the most immersive media [Kaplan-Rakowski and Meseberg 2019] and has been postulated as a first step towards achieving hyperreality, a condition in which consciousness is unable to distinguish reality from a simulation of reality. In its current state, however, VR is far from being truly immersive. Among other shortcomings, the physical confinements of real-world surroundings restrict a user from truly being able to freely move around in the (potentially) infinitely large virtual world, and, as such, developers have opted for alternative means of locomotion. While these artificial techniques achieve the desired result, the sense of presence is damaged in the process, and thus the distinction between the physical and virtual world is exposed.

The solution evaluated in this research is the use of natural walking in a VE designed in such a way as to allow for an infinite amount of virtual space to be traversed within a finite amount of physical space. This is achieved by strategically placing portals throughout the VE (see black doorways in Figure 1(d)) and allowing subspaces within the VE to be overlaid. From a mathematical perspective these are locally Euclidean orbit-manifolds. Users are thus redirected into walking strictly within their play area - creating the illusion that they are exploring an area much larger than it actually is. 


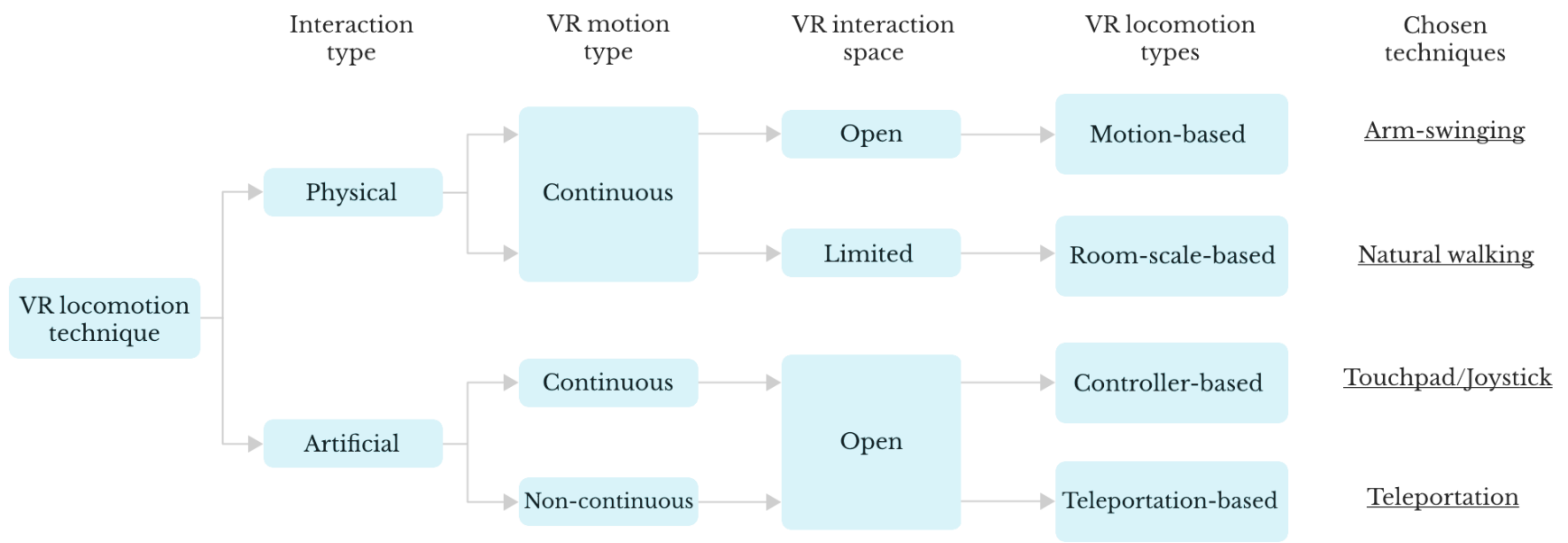

Figure 2: The VR locomotion typology [Boletsis 2017] leading up to the four chosen techniques.

The contribution of this paper lies in determining the viability of this approach as a locomotion technique. To do so we compare it using a quantitative within-subject user experiment $(n=25)$ to three existing techniques:

(1) Teleportation: A teleportation-based technique where users point a ray, originating from their dominant hand, towards a position in the virtual world and instantaneously teleport there once the controller's trigger is pulled.

(2) Arm-swinging: A motion-based technique where users simulate real-life walking by activating both controllers' grips and swinging their arms back and forth, subsequently moving in the direction that they are facing.

(3) Touchpad/foystick: A controller-based technique in which users direct their movement based on input from a 2D-axial control.

These locomotion techniques were selected because they are: frequently adopted in practice, widely tested in existing locomotion experiments and hence useful for benchmarking, and represent a spectrum of approaches since they fall in separate branches of Boletsis' typology (see Figure 2) [Boletsis 2017].

Our user experiment employed both objective and subjective measures. To objectively assess techniques, the time taken for users to complete a set of tasks for a given house (see Figure 1) were recorded and used to evaluate task performance. For subjective assessment, we used questionnaires, including the Simulator Sickness Questionnaire (SSQ) [Kennedy et al. 1993], Game Experience Questionnaire (GEQ) [Poels et al. 2007] and System Usability Scale (SUS) [Peres et al. 2013]. Note that the order in which locomotion techniques were presented to each experiment participant was randomized to mitigate the effects of learning bias.

\section{RELATED WORK}

\subsection{Conventional locomotion techniques}

Due to the importance of locomotion in Virtual Reality, research in this sub-field is abundant.
Of the more prevalent techniques used in real-world applications today, the majority tend to focus less on realism and more on the level of comfort, with a study showing teleportation as being the most widely used due to the lower levels of simulator sickness (SS) it induces [Coomer et al. 2018]. SS is a well-known shortcoming associated with VR experiences, and refers to a subset of motion-induced sickness (MS), with symptoms generally grouped into nausea, oculomotor discomfort, and disorientation [Mourant and Thattacherry 2000]. It occurs when conflicting signals (from a user's eyes, inner ears and/or body tissue sensory receptors) are sent to a user's brain regarding self-movement in a VE [Chang et al. 2020]. In order to mitigate this effect locomotion techniques that more realistically represent a simulation of real-world walking are often used. In one study, the task performance of natural (real walking), semi-natural (based on the VirtuSphere device), and non-natural (based on a video game controller) locomotion techniques were compared [Nabiyouni et al. 2015]. The study found that counter-intuitively, more realism in VR systems does not necessarily correlate with better task performance.

In another instance, a meta-review of 22 locomotion techniques was conducted in order to better understand their impact on users' overall experience, and again it was found that classic, non-natural locomotion techniques outperformed those that simulated natural walking [Cherni et al. 2020].

\subsection{Natural walking (in impossible spaces) as a locomotion technique}

Natural walking is a room scale-based technique whereby a user's physical motion is reflected in the VE and is used as the predominant means of traversal. For this technique, play areas must be large (at least $2 \times 2 \mathrm{~m}$ ), and head-mounted displays (HMDs) must support 6 degrees of freedom $(6 \mathrm{DoF})$ (i.e., both rotational and translational movements are captured). Alongside natural walking, this research evaluates the use of carefully constructed impossible spaces, which are achieved using portals. 
The concept of portals in 3D video games is well-established and showed an initial surge in popularity in 2007 after the release of the game Portal. Another popular title, Antichamber, is infamous among mathematicians for having appropriated the term 'non-Euclidean' in games ${ }^{1}$. Actually, the majority of these experiences take place in 'locally Euclidean orbit-manifolds', where the surgery of worlds changes the topology of its space but not its geometry. Figure 3 from the 'Non-Euclidean Worlds Engine' 2 provides a useful Echeresque illustration of the concept, in which portals (in red) and overlapping spaces (separated on the left and merged on the right) create a space four times larger than it appears.

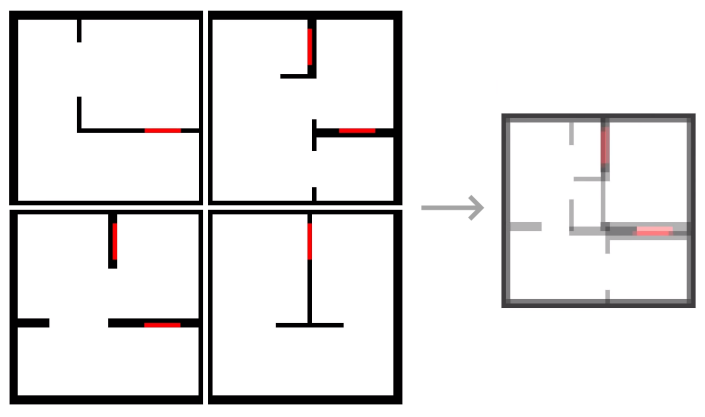

Figure 3: A possible arrangement of four rooms, which have been compressed to fit within a single room.

Since then, several titles (e.g., Spellbound Spire, Tea for God, traVRsal and Unseen Diplomacy) have been developed using natural walking in impossible spaces as the dominant means of locomotion. Even though these examples achieve the end goal of self overlapping architectures, they differ as to the means: either using transitioning areas or portals.

Transitioning areas are intermediate spaces, such as curved corridors [Vasylevska et al. 2013], that allow changes to be made that are invisible to the user. This exploits a phenomenon known as 'change blindness', which refers to a failure to notice changes in a scene that occur outside the subject's field of view [Suma et al. 2011]. It has been determined through perceptual experiments in VR [Suma et al. 2012] that smaller rooms allow for a greater percentage overlap than larger rooms, before users are able to detect that they are in an impossible space. It was also found that these spaces provide a more powerful illusion when users are naïve to the manipulation. In certain cases, where play areas are small or irregular, this approach may be difficult to replicate. To combat this, procedural methods can be used to automatically generate layouts that fit within the tracked space [Vasylevska et al. 2013].

In our research, we accept that users may be aware that they are in an impossible space. This may have consequently lead to lower levels of immersion, however, and negatively affected the scores obtained from the GEQ.

The second more convincing technique, and the one used in our experiment, involves portals. In two separate studies [Freitag et al. 2014; Liu et al. 2018], portals were used as a reorientation technique to redirect users back to the centre of their tracking space once they

\footnotetext{
${ }^{1}$ https://zenorogue.medium.com/non-euclidean-geometry-and-games-fb46989320d4 ${ }^{2}$ https://www.youtube.com/watch?v=kEB11PQ9Eo8
}

had reached a physical boundary. In both cases, it was found that while this method allowed for a better utilization of the available space and did not cause additional SS, it did lead to longer completion times for the given tasks. Unlike in our experiment, however, measures such as immersion and usability were not considered.

\section{DESIGN AND IMPLEMENTATION}

The Unity game engine was used due to its ease of use and flexibility in switching between platforms (i.e., Android - Oculus Quest and Windows - Oculus Rift and HTC Vive). Unfortunately, because of the use of some proprietary assets in the experiment, we are unable to release the entire source code. Nevertheless, our VR portal implementation, PortalsVR ${ }^{3}$, is freely available.

\subsection{Locomotion techniques}

The Unity Extended Reality (XR) interaction toolkit was used as a foundation for the implementation of the conventional locomotion techniques. While teleportation is supported in this package by default, the others required further implementation.

Touchpad/joystick movement was determined based on input from the 2D-axis of the user's dominant controller, with viewer orientation used as a frame of reference. Arm-swinging movement was determined in two parts: speed of movement was based on the distance both controllers moved in the previous frame (with respect to the player), while movement direction was based solely on the user's orientation.

As discussed previously, natural walking is included by default for HMDs that support room-scale VR. This research, therefore, focuses on its application in impossible spaces, achieved using portals. As a base, we made use of an existing single viewpoint implementation of portals ${ }^{4}$ with the following adaptations:

(1) Stereoscopic Rendering: The view through a portal should align with what the user would see if they were transformed to a position and rotation with respect to the destination portal equivalent to their position and rotation with respect to the source portal. In order to correctly portray the view through a portal in VR, different images of the subspace beyond the portal need to be projected as textures onto the portal surface for each eye. For this, portals each required an additional camera and screen. Screens were then assigned to separate layers, and the users' eyes culled so as to only view one screen per eye. The left and right portal cameras could then render separate views for each eye, while tracking the position and rotation of an alias for each eye. The additional camera and projected image per portal does impact performance. This is partially compensated for using frustum culling and occlusion to determine whether a portal needs rendering.

(2) Teleportation: A pair of portals should allow users to travel instantaneously between them. In our case, portal pairs always have the same local position and rotation with respect to the physical room. Thus, the user's rotation is not altered when teleporting, and so travel is achieved by simply offsetting the position of the player to the destination subspace.

\footnotetext{
${ }^{3}$ https://github.com/daniellochner/PortalsVR under the MIT license

${ }^{4}$ https://github.com/SebLague/Portals
} 
(3) Occlusion: Objects that fall in front of the portal surface (i.e., between the viewpoint and the portal) must not be rendered in the portal display. This is achieved by setting the camera's near clipping plane to coincide with the portal surface.

(4) Clipping: Unfortunately, because the near clipping plane cannot be set to zero, a portal may be clipped away when the viewpoint approaches too closely. To overcome this, the portal surface is dynamically deformed to mould around the player's head. This, however, allows objects on the other side of a portal to clip through. A solution is to include an array of clipped objects, which deactivate when behind the player. Another clipping issue occurs when the player's avatar crosses the portal, specifically when the stereoscopic camera rig is on one side of a portal and models of the controllers or the clipboard are on the other. Because this is only a single player proof of concept, this can be circumvented by introducing an overlay camera, which ensures that these models are always rendered in front of other views.

We have not yet touched on the challenges posed by the layout design of impossible spaces. This process is significantly more constrained than conventional level design: individual sub-spaces can be no larger than the physical space and portals must be carefully positioned and oriented. Providing authoring tools specific to impossible spaces would be a worthwhile endeavour, but is beyond the scope of this work.

\subsection{Experiment}

In order to separately evaluate each of the four locomotion techniques, four distinct house environments (see Figure 1) were constructed. This was necessary as the design of an impossible space requires careful consideration of portal placement. The choice to then share a single other environment between the remaining three conventional techniques would have damaged experimental validity, as it would introduce a potential learning effect not afforded to natural walking.

While different in architecture, the houses were designed to be the same size (i.e., $100 \mathrm{~m}^{2}$ ). In the case of natural walking, however, it was necessary to scale down on the horizontal XZ-plane to as little as $25 \%$ of the original size (i.e., rooms ranged from $5 \times 5 \mathrm{~m}$ to a minimum of $2.5 \times 2.5 \mathrm{~m}$ ) to ensure that the VE matched a user's play area. This was necessary as many of the subjects had limited physical space. Ideally, this could have been avoided by conducting in-person experiments in a single space with a consistent setup, but the COVID-19 pandemic made this impossible. It was, therefore, necessary that instructions for the setup and running of the experiment be as intuitive and easy to perform as possible.

To achieve this tutorials were provided for all the locomotion technique. These included a list of steps that explained how to use each locomotion technique, with each step needing to be "checked off" before proceeding to the next. After each technique was explained, users then had to apply their knowledge by moving to a target position. After this, the user performed a set of tasks. Because the process for each evaluation was the same (i.e., users had a set of tasks to complete for each house), viewing the clipboard as well as interacting with objects and completing tasks was explained once in a separate tutorial at the beginning of the experiment.
Table 1: The different (but functionally equivalent) tasks chosen for each of the four locomotion techniques.

\begin{tabular}{clll}
\hline & \multicolumn{1}{c}{ Task 1 } & \multicolumn{1}{c}{ Task 2 } & \multicolumn{1}{c}{ Task 3 } \\
\hline $\mathrm{T}$ & Turn on lights & Boil kettle & Set alarm \\
$\mathrm{T} / \mathrm{J}$ & Empty bins & Wash dishes & Set alarm \\
AS & Fix wall plugs & Watch TV & Set alarm \\
NW & Collect towels & Light fireplace & Set alarm \\
\hline
\end{tabular}

While tasks differed for the sake of variety (see Table 1), the amount of work required to complete all tasks for a given house was designed to be the same.

The first task (exploration phase) comprised multiple actions that were dispersed to ensure users moved around the entire house. The second was positioned at a single location, and finally, the third was, in all cases, to return to the starting position and "set the alarm", which was symbolic of leaving the house. To further ensure equivalence, actions could be completed with a single button press, and each house had three tasks - two unique and one similar (see Figure 4). As an affordance in identifying tasks, interactable objects were outlined in white when in view and green when touched. On task completion, feedback was provided to users using a sound and/or particle effect. Individual subtask actions were also incremented on the user's clipboard until the task was fully complete, after which a line was drawn through the task.

Several usability improvements were incorporated into the experiment, including: displaying users' exact play area dimensions on their monitors (required for completion of the on-line form), allowing users to choose their dominant hand, displaying the correct controller model (a hand when the device was unknown) and prompting users to move back to the centre of their physical play area if they had accidentally moved during an evaluation.

Some trade-offs were necessary to ensure adequate performance. The environment was designed using a low poly asset pack, which made use of a single material and a texture atlas to reduce the number of draw calls. Colliders were also removed from models where possible to reduce load times. Finally, shadows were disabled, and dynamic lighting substituted with ambient light baked into textures combined with a single directional light to allow for some visual differentiation between similar objects.

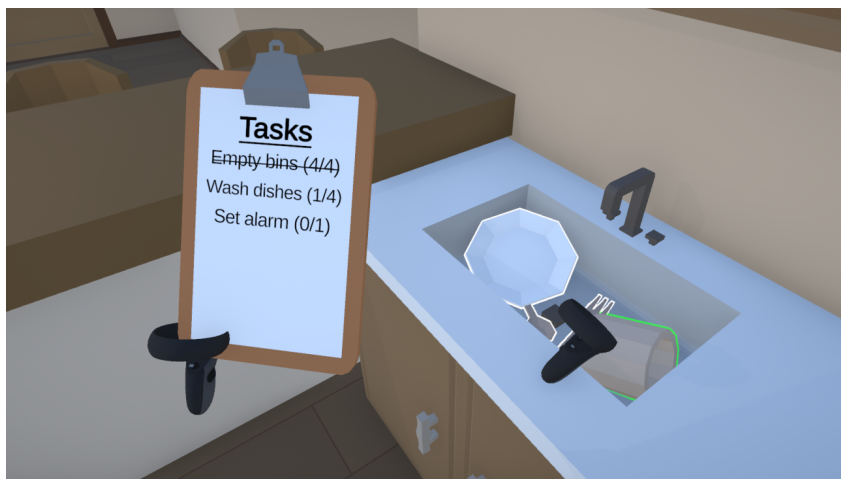

Figure 4: Clipboard with the next task being to wash dishes. 


\section{PROCEDURES}

The following automated protocol was applied for all iterations of the experiment. To begin, subjects signed a participation consent form that detailed the aims, procedures, and potential risks and benefits of participation. Participants were allowed to withdraw from the study at any time without prejudice. They then provided some demographic information, after which they donned their HMDs and continued with the VE tasks.

First, an initial interactive VE tutorial was provided to familiarise users with object interaction and the use of a clipboard to guide task completion. Subsequently, participants were placed in their first randomly-assigned house, and introduced to the associated locomotion technique through an interactive tutorial (see Figure 5). In all cases, the technique's controls were shown first, and then a target was positioned in the world (with tutorial dialog still active), which users had to reach. This ensured that they knew how to traverse the VE before proceeding with the evaluation. After completing all tasks on their clipboards, users were then asked to fill in a set of three questionnaires with instructions shown under each heading. For those questions that included technical terminology, descriptions were also provided in layman's terms. After submitting, users then returned to the simulation and repeated this process for the other three locomotion techniques.

After each environment, participants completed an SSQ, GEQ, and SUS questionnaire. Instead of using the core version of the GEQ, however, the in-game version (iGEQ) was used due to time constraints. These specific questionnaires were chosen based on their prevalence in VR research [Boletsis and Cedergren 2019; Bozgeyikli et al. 2016; Coomer et al. 2018], and due to the significance of the measures they test for.

For instance, locomotion is known to be a potential source of simulator sickness because it can cause perceptual conflict [Saredakis et al. 2020]. Likewise, it is important to test the immersion and easeof-use of any VE interaction method. Finally, if a VR application is task oriented then efficiency-of-use is likely to be a consideration.

Once complete, task performance times were shown and the participants were required to manually enter these into the form, before submitting and notifying the experimenters of completion. After their results were confirmed to be in the database, they were thanked and remunerated (\$10) for their participation.

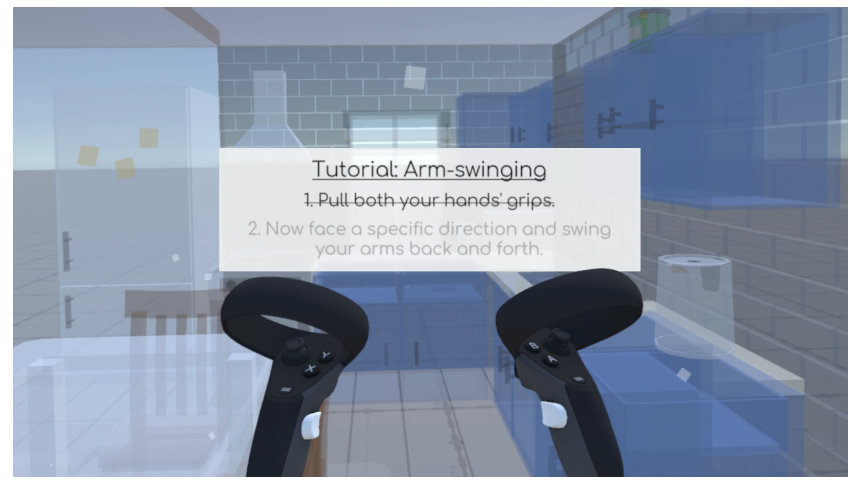

Figure 5: Tutorial used to explain how to move using the arm-swinging locomotion technique.

\section{DATA ANALYSIS AND RESULTS}

Participants were recruited through private messaging and announcements on several social media platforms. Of the 25 participants who took part, 10 conducted the experiment in-person with the experimenter's setup and 15 conducted it at home with their own VR equipment. This approach was necessitated by the exigencies of the COVID-19 pandemic.

In order to ensure consistent treatment of all participants, little to no additional assistance was provided to those who conducted the experiment in-person. In total, 300 questionnaires and 96 timings were considered in this study, with one user's times excluded because they needed to re-arrange furniture within their play area while performing the experiment. Non-intrusive demographics were captured through the on-line form to help provide insight into variation between results. In terms of frequency of VR usage, participants rated themselves on a Likert scale from 1 (not very often) to 5 (very often). The majority reported that they did not use VR very often, with $36 \%$ reporting a $1,8 \%$ a $2,16 \%$ a $3,24 \%$ a 4 and $16 \%$ a 5 . For age, $32 \%$ responded that they were $<20,20 \%$ in the range $20-25,8 \%$ in the range $25-30$ and $40 \%>30$. Since users were required to move around, we asked whether or not a user's HMD was tethered, as well as the dimensions of their play area (which were provided to them in-game). It was noted that $48 \%$ used tethered and 52\% untethered headsets, and that the average play area was $8.42 \mathrm{~m}^{2}$ (ranging between 6.25 and $16.00 \mathrm{~m}^{2}$ ).

In analysing our continuous measures (game experience, simulator sickness, system usability and task performance), we applied the Shapiro-Wilks test of normality on the residuals. In cases where we found with statistical significance that residuals followed a normal distribution, a repeated-measures ANOVA was performed to determine whether a significant difference between techniques existed. Otherwise, normality was not assumed, and the Friedman test was applied. In cases where a significant difference was found, we followed up with a post-hoc Tukey's HSD test (for normal data) or a Wilcoxon matched pair signed rank sum test (for non-parametric data), both with Bonferroni correction, to determine which particular treatments differed. This is necessary, as multiple analyses need to be performed on the same sample of data (where familywise type 1 error rate is known to be larger than per analysis error rate). Note that in all tests a significance level of $\alpha=0.05$ was adopted.

\subsection{Simulator Sickness}

Table 2 and Figure 6 show SSQ results as tabulated means and standard deviations, and box-and-whisker diagrams, respectively. In analysing SSQ data, none of the symptoms passed the test of normality, and so we proceeded with the Friedman test, where it was noted that a significant difference existed between treatments for nausea $\left(\chi^{2}=18.238, p<0.001\right)$, oculomotor discomfort $\left(\chi^{2}=\right.$ $19.618, p<0.001)$, disorientation $\left(\chi^{2}=20.754, p<0.001\right)$ and total severity $\left(\chi^{2}=19.875, p<0.001\right)$ (i.e., all symptoms).

Table 3 shows the results of post-hoc tests of differences between techniques for symptoms of the SSQ where significant differences were found. 
Table 2: Means and standard deviations for scores from the SSQ.

\begin{tabular}{|c|c|c|c|c|c|c|c|c|}
\hline & \multicolumn{2}{|c|}{ Nausea } & \multicolumn{2}{|c|}{ Oculomotor } & \multicolumn{2}{|c|}{ Disorientation } & \multicolumn{2}{|c|}{ Tot. Severity } \\
\hline & $\bar{x}$ & $s$ & $\bar{x}$ & $s$ & $\bar{x}$ & $s$ & $\bar{x}$ & $s$ \\
\hline $\mathrm{T}$ & 11.45 & 18.82 & 10.69 & 18.00 & 17.96 & 31.10 & 14.59 & 23.81 \\
\hline $\mathrm{T} / \mathrm{J}$ & 40.93 & 39.81 & 34.34 & 38.40 & 60.13 & 62.44 & 49.14 & 49.27 \\
\hline AS & 30.34 & 32.22 & 24.10 & 25.49 & 32.99 & 42.48 & 32.65 & 34.85 \\
\hline NW & 21.46 & 29.86 & 17.28 & 22.77 & 29.65 & 44.05 & 24.91 & 33.55 \\
\hline
\end{tabular}

Table 3: Post-hoc between-technique test results for scores from the SSQ.

\begin{tabular}{|c|c|c|c|c|c|c|c|c|}
\hline & \multicolumn{2}{|c|}{ Nausea } & \multicolumn{2}{|c|}{ Oculomotor } & \multicolumn{2}{|c|}{ Disorientation } & \multicolumn{2}{|c|}{ Tot. Severity } \\
\hline & $p$ & $r$ & $p$ & $r$ & $p$ & $r$ & $p$ & $r$ \\
\hline $\mathrm{T}-\mathrm{T} / \mathrm{J}$ & 0.003 & 0.402 & 0.002 & 0.462 & 0.002 & 0.517 & 0.001 & 0.469 \\
\hline $\mathrm{T}-\mathrm{AS}$ & 0.007 & 0.649 & 0.024 & 0.527 & & & 0.011 & 0.605 \\
\hline $\mathrm{T} / \mathrm{J}-\mathrm{AS}$ & & & & & 0.044 & 0.621 & & \\
\hline
\end{tabular}

Figure 6: Box-and-whisker diagram for scores from the 4 symptoms of the SSQ.

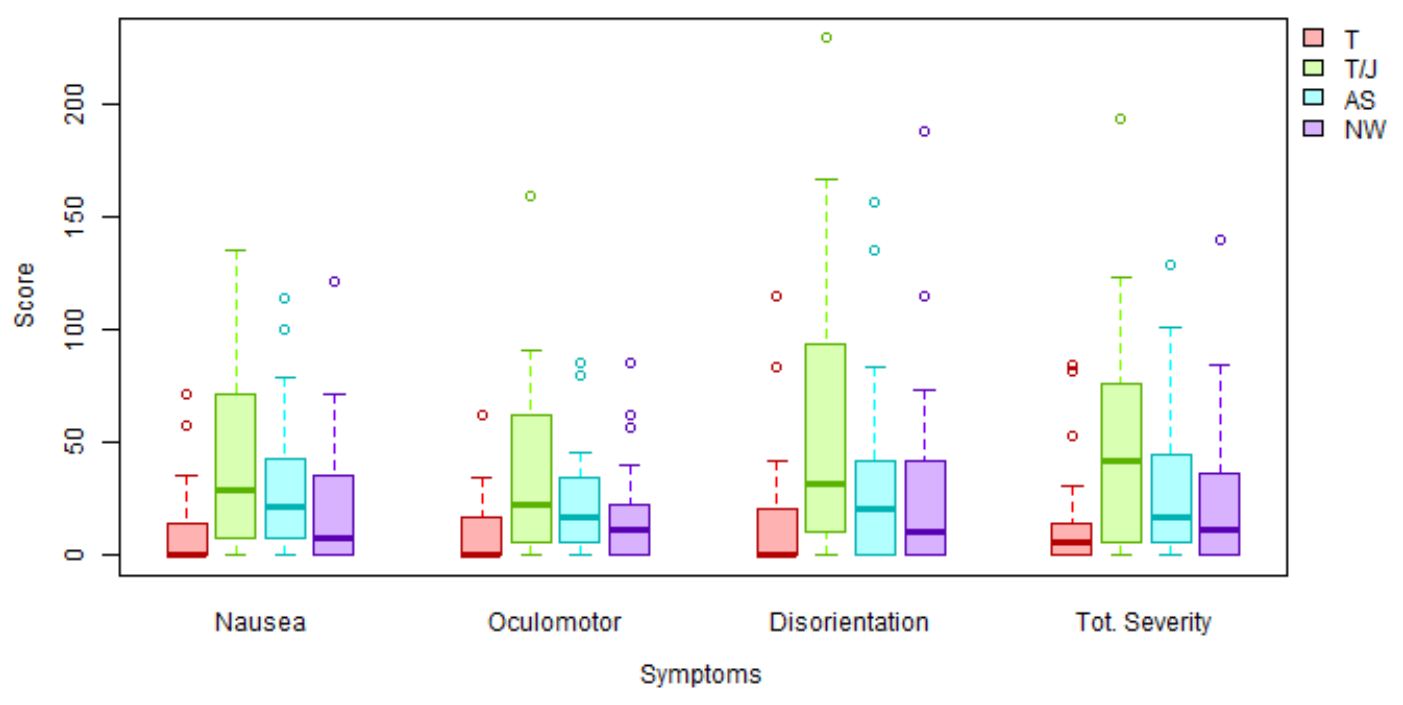

\subsection{Game Experience}

Table 4 and Figure 7 show GEQ results as tabulated means and standard deviations, and box-and-whisker diagrams, respectively. In analysing GEQ data, competence, challenge and positive affect passed the test of normality and so we proceeded to within-subjects ANOVA. On the other hand, immersion, flow, tension and negative affect did not follow a normal distribution and so the Friedman test was applied. It was found that a significant difference existed between techniques for immersion $\left(\chi^{2}=28.347, p<0.001\right)$, flow $\left(\chi^{2}=9.723, p=0.021\right)$, challenge $(F=9.377, p<0.001)$, negative affect $\left(\chi^{2}=10.399, p=0.015\right)$ and positive affect $(F=3.094, p=$ 0.032 ), but not competence and tension. While a Friedman test on flow indicated significant differences among techniques, performing a Wilcoxon signed rank sum test on all combinations led to none.

Table 5 shows the results of post-hoc tests for differences between techniques on the dimensions of the GEQ, where significance was found.

\subsection{System Usability}

Table 6 and Figure 8 show SUS results as tabulated means and standard deviations, and box-and-whisker diagrams, respectively. In analysing SUS scores, a Friedman test was used due to residuals not following a normal distribution, where a significant difference was identified between techniques $\left(\chi^{2}=15.637, p=0.001\right)$. Table 7 shows the results of post-hoc tests of differences between techniques.

The theoretical average of SUS scores is recorded as 68 (based on 500 prior studies ${ }^{5}$ ), and a student's T-test was thus performed analysing the significance of recorded means. It was noted that teleportation $(T=5.678, p<0.001)$, touchpad/joystick $(T=1.919, p=$ $0.03)$ and natural walking $(T=5.252, p<0.001)$ obtained scores significantly above the average SUS score, however arm-swinging did not.

$\overline{5}$ Jeff Sauro. (2011). Measuring Usability with the System Usability Scale (SUS). https://measuringu.com/sus/ 
Table 4: Means and standard deviations for scores from the GEQ.

\begin{tabular}{|c|c|c|c|c|c|c|c|c|c|c|c|c|c|c|}
\hline & \multicolumn{2}{|c|}{ Competence } & \multicolumn{2}{|c|}{ Immersion } & \multicolumn{2}{|c|}{ Flow } & \multicolumn{2}{|c|}{ Tension } & \multicolumn{2}{|c|}{ Challenge } & \multicolumn{2}{|c|}{ Neg. Affect } & \multicolumn{2}{|c|}{ Pos. Affect } \\
\hline & $\bar{x}$ & $s$ & $\bar{x}$ & $s$ & $\bar{x}$ & $s$ & $\bar{x}$ & $s$ & $\bar{x}$ & $s$ & $\bar{x}$ & $s$ & $\bar{x}$ & $s$ \\
\hline $\mathrm{T}$ & 3.14 & 1.10 & 2.82 & 1.32 & 2.86 & 1.26 & 1.46 & 0.61 & 2.16 & 0.86 & 1.90 & 0.87 & 3.30 & 0.99 \\
\hline $\mathrm{T} / \mathrm{J}$ & 3.20 & 1.02 & 2.90 & 1.22 & 2.86 & 1.23 & 1.52 & 0.94 & 2.26 & 0.82 & 1.96 & 1.01 & 3.16 & 1.16 \\
\hline AS & 3.32 & 0.97 & 3.28 & 1.22 & 3.16 & 1.14 & 1.52 & 0.67 & 2.92 & 0.93 & 2.08 & 0.92 & 3.34 & 0.99 \\
\hline NW & 3.44 & 1.07 & 3.70 & 1.13 & 3.56 & 1.21 & 1.48 & 0.74 & 2.96 & 1.12 & 1.58 & 0.70 & 3.76 & 1.03 \\
\hline
\end{tabular}

Table 5: Post-hoc between-technique test results for scores from the GEQ.

\begin{tabular}{|c|c|c|c|c|c|c|c|c|}
\hline & \multicolumn{2}{|c|}{ Immersion } & \multicolumn{2}{|c|}{ Challenge } & \multicolumn{2}{|c|}{ Neg. Affect } & \multicolumn{2}{|c|}{ Pos. Affect } \\
\hline & $p$ & $r$ & $p$ & $r$ & $p$ & $r$ & $p$ & $r$ \\
\hline $\mathrm{T}-\mathrm{AS}$ & 0.020 & 0.849 & $<0.001$ & 0.760 & & & & \\
\hline $\mathrm{T}-\mathrm{NW}$ & 0.003 & 0.724 & $<0.001$ & 0.800 & & & & \\
\hline $\mathrm{T} / \mathrm{J}-\mathrm{AS}$ & & & 0.004 & 0.660 & & & & \\
\hline $\mathrm{T} / \mathrm{J}-\mathrm{NW}$ & $<0.001$ & 0.800 & 0.002 & 0.700 & & & 0.020 & 0.600 \\
\hline AS - NW & 0.039 & 0.831 & & & 0.018 & 0.634 & & \\
\hline
\end{tabular}

Figure 7: Box-and-whisker diagram for scores from the 7 dimensions of the GEQ.

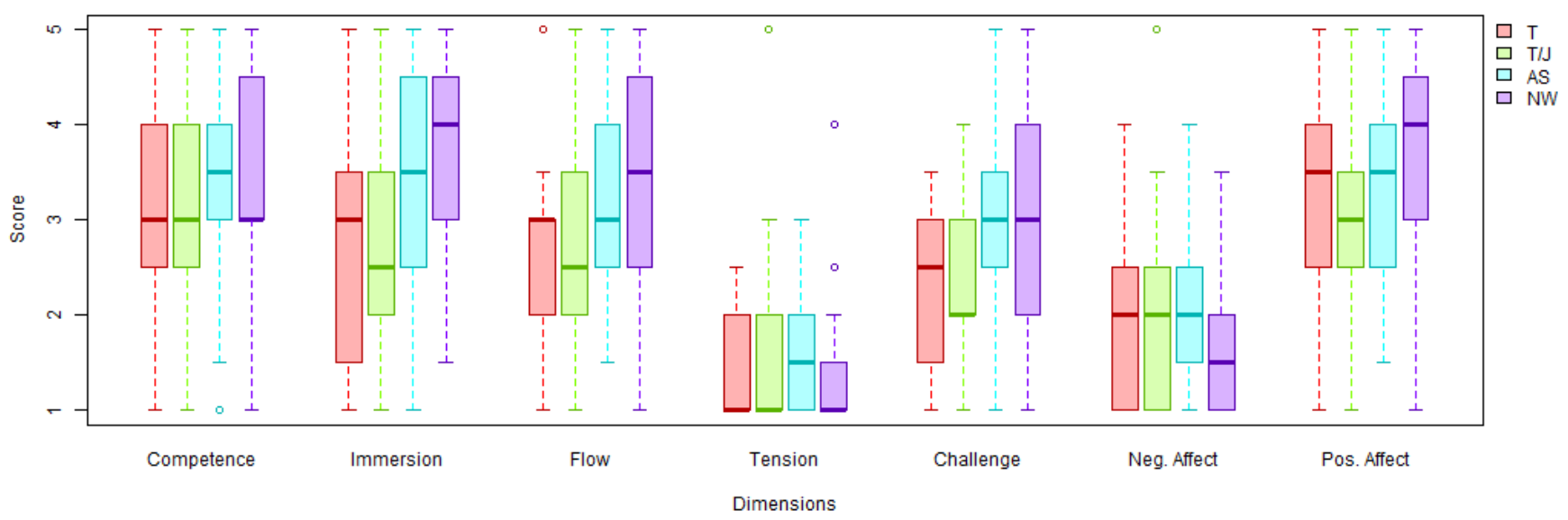

Table 6: Means and standard deviations from the SUS.

\begin{tabular}{ccc}
\hline & $\bar{x}$ & $s$ \\
\hline $\mathrm{T}$ & 81.80 & 12.15 \\
$\mathrm{~T} / \mathrm{J}$ & 74.90 & 17.98 \\
$\mathrm{AS}$ & 72.70 & 17.59 \\
$\mathrm{NW}$ & 84.70 & 15.90 \\
\hline
\end{tabular}

Table 7: Post-hoc between-technique test results for scores from the SUS.

\begin{tabular}{ccc}
\hline & $p$ & $r$ \\
\hline T/J - NW & 0.018 & 0.387 \\
AS - NW & 0.010 & 0.518 \\
\hline
\end{tabular}

Figure 8: Box-and-whisker diagram for scores from the SUS.

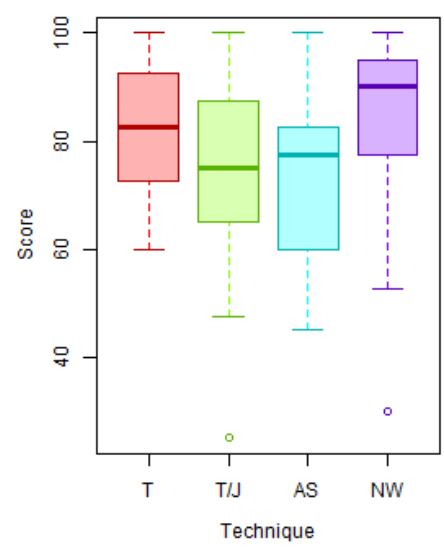


Table 8: Means and standard deviations for completion times (in seconds).

\begin{tabular}{ccc}
\hline & $\bar{x}$ & $s$ \\
\hline $\mathrm{T}$ & 121.56 & 91.76 \\
$\mathrm{~T} / \mathrm{J}$ & 126.82 & 73.10 \\
$\mathrm{AS}$ & 213.99 & 160.63 \\
$\mathrm{NW}$ & 173.15 & 144.06 \\
\hline
\end{tabular}

Table 9: Post-hoc between-technique tests for completion times.

\begin{tabular}{ccc}
\hline & $p$ & $r$ \\
\hline $\mathrm{T}-\mathrm{AS}$ & $<0.001$ & 0.822 \\
$\mathrm{~T} / \mathrm{J}-\mathrm{AS}$ & 0.006 & 0.185 \\
\hline
\end{tabular}

Figure 9: Box-and-whisker diagram for completion times.

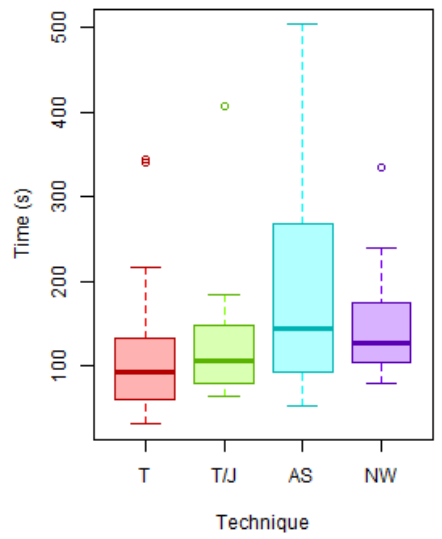

\subsection{Task Performance}

Table 8 and Figure 9 show completion times as tabulated means and standard deviations, and box-and-whisker diagrams, respectively, for task completion times (in seconds). In analysing times, a Friedman test was used since residuals did not follow a normal distribution, and a significant difference was identified between techniques $\left(\chi^{2}=24.967, p<0.001\right)$. Table 9 shows the significant results of a post-hoc test of difference between techniques.

\section{DISCUSSION}

In our experiments, natural walking (NW) in impossible spaces evinces selected improvement in game experience over other techniques. Specifically, participants experienced significantly less negative affect and greater positive affect when using NW over armswinging (AS) and touchpad/joystick $(\mathrm{T} / \mathrm{J})$, respectively. Importantly, participants felt significantly more immersed and challenged with NW than either teleportation (T) or $\mathrm{T} / \mathrm{J}$. The latter is not a negative outcome because, if utilized correctly, some level of challenge is known to be a large factor in remedying boredom [Martin et al. 2006]. With regards to SS, in examining the means, NW consistently placed better than $\mathrm{T} / \mathrm{J}$ and AS, but worse than T. However, none of these NW results were significant. What was significant is the greater SS induced by AS and T/J as compared to T. We note, that this is consistent with other studies in VR locomotion [Boletsis and Cedergren 2019; Bond and Nyblom 2019]. The lower levels of SS for $\mathrm{T}$ can be attributed to the fact that signals to users' brains regarding self-movement do not conflict, as users are instantaneously transported to locations in the VE. On the other hand, this lacks realism and can lead to lower levels of immersion, as supported by the $T$ results for the immersion item of the GEQ.

In terms of system usability, NW achieved the highest mean scores on the SUS. This improvement was significant $(\alpha=0.05)$ for NW as compared to T/J and AS, but not T. This outcome should be qualified, however, by the high number of participants unfamiliar with VR controllers and the functionality of their different buttons.

On the other hand, NW saw no significant reductions in task performance over the other techniques. It was hypothesized that task completion time may have reduced due to users with lower levels of spatial awareness becoming disoriented and consequently lost in the VE during NW [Rasheed et al. 2015], however, no significant results were obtained with regard to NW and disorientation for scores from the SSQ.

We also provided an opportunity for participants to provide qualitative feedback. As anticipated, four participants mentioned that they bumped into real-world obstacles while using NW and that this disrupted their sense of presence. With regards to implementation, three experienced VR users suggested including snap-rotation (using the non-dominant controller's joystick) for additional turning, and two stated that they would rather have used their non-dominant hand to move when using $\mathrm{T} / \mathrm{J}$. One participant also mentioned that T/J was better implemented in Half-Life: Alyx, but this may be attributed to additional factors, as a study comparing the same techniques received similar results to ours when comparing $\mathrm{T}$ and AS [Bond and Nyblom 2019].

\subsection{Limitations}

Some participants were hamstrung by play areas that did not match the ideal specification. While we automatically resized the impossible space to compensate, this is a possible confounding factor in the experiment and may have led to lower levels of game experience.

Teleportation was identified by some participants as being 'too fast', which could be avoided by introducing a fade-to-black or cool-down mechanism. Tasks could also have been randomized between houses to better correct for any inadvertent correlations between task and locomotion technique (e.g., switching on lights compared to bending over to empty bins). Finally, it would have been worthwhile recording some additional information for remote experiments, such as average rendered frames per second and specific make of HMD (e.g., Oculus Rift, Oculus Quest, HTC Vive, etc.), as these might have been confounding factors.

In summary, conducting experiments remotely perforce meant a less controlled environment, with potential variation in equipment, play area and the extent of outside interruptions. Uniform and controlled laboratory conditions might have lead to more marked and significant differences between treatments. Nevertheless, this does improve the ecological validity of our results in the sense that typical variations in real-world use are factored into the experiment. 


\section{CONCLUSION}

In this study, we undertook a quantitative evaluation of four VR locomotion techniques to assess the viability of natural walking when used in impossible spaces as compared to the established methods of teleportation, touchpad/joystick, and arm-swinging. The continuous measures used in our experiment included game experience, simulator sickness, system usability and task performance, which were evaluated through the use of the GEQ, SSQ, and SUS questionnaires and recorded task completion times. In this study, natural walking shows statistically significant improvement in immersion (over teleportation and touchpad/joystick) and system usability (over arm-swinging and touchpad/joystick), but at the expense of task performance (when compared to teleportation and touchpad/joystick, specifically). For simulator sickness the difference between teleportation and natural walking was inconclusive (unlike the significant improvement of teleportation over both touchpad/joystick and arm-swinging).

In summary, natural walking in impossible spaces shows promise as a VR locomotion technique because it strikes a balance between immersion, usability, and relatively low levels of simulator sickness. One caveat, however, is the difficulty of constructing impossible spaces and their restriction to representing indoor environments with VR subspaces that are no larger than the physical play area.

\section{ACKNOWLEDGMENTS}

This work is based on the research supported wholly by the National Research Foundation of South Africa (Grant Number: 129257).

\section{REFERENCES}

Costas Boletsis. 2017. The New Era of Virtual Reality Locomotion: A Systematic Literature Review of Techniques and a Proposed Typology. Multimodal Technologies and Interaction 1, 4 (2017). https://doi.org/10.3390/mti1040024

Costas Boletsis and Jarl Erik Cedergren. 2019. VR Locomotion in the New Era of Virtual Reality: An Empirical Comparison of Prevalent Techniques. Advances in Human-Computer Interaction 2019 (2019), 15. https://doi.org/10.1155/2019/7420781

D. Bond and M. Nyblom. 2019. Evaluation of four different virtual locomotion techniques in an interactive environment. Technical Report. Blekinge Institute of Technology.

Evren Bozgeyikli, Andrew Raij, Srinivas Katkoori, and Rajiv Dubey. 2016. Point and Teleport Locomotion Technique for Virtual Reality. In Proceedings of the 2016 Annual Symposium on Computer-Human Interaction in Play (Austin, Texas, USA) (CHI PLAY '16). Association for Computing Machinery, New York, NY, USA, 205-216. https://doi.org/10.1145/2967934.2968105

Eunhee Chang, Hyun Taek Kim, and Byounghyun Yoo. 2020. Virtual Reality Sickness: A Review of Causes and Measurements. International fournal of Human-Computer Interaction 36, 17 (2020), 1658-1682. https://doi.org/10.1080/10447318.2020.1778351

Heni Cherni, Natacha Métayer, and Nicolas Souliman. 2020. Literature review of locomotion techniques in virtual reality. International fournal of Virtual Reality 20 1 (Mar. 2020), 1-20. https://doi.org/10.20870/IJVR.2020.20.1.3183

Noah Coomer, Sadler Bullard, William Clinton, and Betsy Williams-Sanders. 2018 Evaluating the Effects of Four VR Locomotion Methods: Joystick, Arm-Cycling, Point-Tugging, and Teleporting. In Proceedings of the 15th ACM Symposium on Applied Perception (Vancouver, British Columbia, Canada) (SAP '18). Association for Computing Machinery, New York, NY, USA, Article 7, 8 pages. https://doi.org/ $10.1145 / 3225153.3225175$

Sebastian Freitag, Dominik Rausch, and Torsten Kuhlen. 2014. Reorientation in virtual environments using interactive portals. In 2014 IEEE Symposium on $3 D$ User Interfaces (3DUI). 119-122. https://doi.org/10.1109/3DUI.2014.6798852

Regina Kaplan-Rakowski and Kay Meseberg. 2019. Immersive Media and Their Future Springer International Publishing, Cham, 143-153. https://doi.org/10.1007/978-3030-27986-8_13

Robert S. Kennedy, Norman E. Lane, Kevin S. Berbaum, and Michael G. Lilienthal 1993. Simulator Sickness Questionnaire: An Enhanced Method for Quantifying Simulator Sickness. The International fournal of Aviation Psychology 3, 3 (1993), 203-220. https://doi.org/10.1207/s15327108ijap0303 3

James Liu, Hirav Parekh, Majed Al-Zayer, and Eelke Folmer. 2018. Increasing Walking in VR Using Redirected Teleportation. In Proceedings of the 31st Annual ACM
Symposium on User Interface Software and Technology (Berlin, Germany) (UIST '18). Association for Computing Machinery, New York, NY, USA, 521-529. https: //doi.org/10.1145/3242587.3242601

Marion Martin, Gaynor Sadlo, and Graham Stew. 2006. The phenomenon of boredom. Qualitative Research in Psychology 3, 3 (2006), 193-211. https://doi.org/10.1191/ 1478088706qrp066oa

Ronald R. Mourant and Thara R. Thattacherry. 2000. Simulator Sickness in a Virtual Environments Driving Simulator. Proceedings of the Human Factors and Ergonomics Society Annual Meeting 44, 5 (2000), 534-537. https://doi.org/10.1177/ 154193120004400513

Mahdi Nabiyouni, Ayshwarya Saktheeswaran, Doug A. Bowman, and Ambika Karanth. 2015. Comparing the performance of natural, semi-natural, and non-natural locomotion techniques in virtual reality. In 2015 IEEE Symposium on 3D User Interfaces (3DUI). 3-10. https://doi.org/10.1109/3DUI.2015.7131717

S. Camille Peres, Tri Pham, and Ronald Phillips. 2013. Validation of the System Usability Scale (SUS): SUS in the Wild. Proceedings of the Human Factors and Ergonomics Society Annual Meeting 57, 1 (2013), 192-196. https://doi.org/10.1177/ 1541931213571043

K. Poels, Y.A.W. de Kort, and W.A. IJsselsteijn. 2007. Game Experience Questionnaire: development of a self-report measure to assess the psychological impact of digital games. Technische Universiteit Eindhoven.

Fabin Rasheed, Prasad Onkar, and Marisha Narula. 2015. Immersive Virtual Reality to Enhance the Spatial Awareness of Students. In Proceedings of the 7th International Conference on HCI, IndiaHCI 2015 (Guwahati, India) (IndiaHCI'15). Association for Computing Machinery, New York, NY, USA, 154-160. https://doi.org/10.1145/ 2835966.2836288

Dimitrios Saredakis, Ancret Szpak, Brandon Birckhead, Hannah A. D. Keage, Albert Rizzo, and Tobias Loetscher. 2020. Factors Associated With Virtual Reality Sickness in Head-Mounted Displays: A Systematic Review and Meta-Analysis. Frontiers in Human Neuroscience 14 (2020), 96. https://doi.org/10.3389/fnhum.2020.00096

Evan A. Suma, Seth Clark, David Krum, Samantha Finkelstein, Mark Bolas, and Zachary Warte. 2011. Leveraging change blindness for redirection in virtual environments. In VR 2011 - IEEE Virtual Reality 2011, Proceedings (Proceedings - IEEE Virtual Reality). 159-166. https://doi.org/10.1109/VR.2011.5759455 18th IEEE Virtual Reality Conference, VR 2011 ; Conference date: 19-03-2011 Through 23-03-2011.

Evan A. Suma, Zachary Lipps, Samantha Finkelstein, David M. Krum, and Mark Bolas. 2012. Impossible Spaces: Maximizing Natural Walking in Virtual Environments with Self-Overlapping Architecture. IEEE Transactions on Visualization and Computer Graphics 18, 4 (2012), 555-564. https://doi.org/10.1109/TVCG.2012.47

Khrystyna Vasylevska, Hannes Kaufmann, Mark Bolas, and Evan A. Suma. 2013. Flexible spaces: A virtual step outside of reality. In 2013 IEEE Virtual Reality (VR). 109-110. https://doi.org/10.1109/VR.2013.6549386 